\title{
ANÁLISE DO COMPORTAMENTO HIDRODINÂMICO EM UMA ETA DE CICLO COMPLETO
}

\author{
Ana Christina Horner Silveira ${ }^{1}$
}

Rafael Montanhini Soares de Oliveira ${ }^{2}$

\section{Sandra Medina Benini ${ }^{3}$}

Resumo: Tendo em vista a importância dos estudos hidrodinâmicos em estações de tratamento de água, o presente trabalho buscou estudar o funcionamento da Estação de tratamento de água ETA 006 na cidade de Palmas-TO. A referida ETA é operada pela Companhia de Saneamento do Tocantins - SANEATINS e está em operação desde 1999. A tecnologia de tratamento é de ciclo completo e funciona atualmente com uma vazão de $500 \mathrm{~L} / \mathrm{s}$ tendo capacidade total para $700 \mathrm{~L} / \mathrm{s}$. A estação é responsável pela maior parte do abastecimento de Palmas, atendendo mais de $60 \%$ da população. A água tratada na ETA 006 é captada no ribeirão Taquarussu, um pequeno manancial cuja nascente fica dentro do município de Palmas. Porém, a vazão do manancial nos últimos anos vem diminuindo consideravelmente, fazendo com que a Companhia de Saneamento avalie outra de fonte de abastecimento para Palmas. Assim, devido à necessidade de reforma/ampliação da estação, e uma possível mudança de captação da água bruta, foi realizado o estudo hidrodinâmico na ETA 006, avaliando principalmente o funcionamento das unidades de floculação e decantação.

\footnotetext{
${ }^{1}$ Engenheira Civil pela UNISINOS, Especialista em Saneamento Ambiental e Mestranda em Engenharia Ambiental pela UFT, Engenheira da Companhia de Saneamento do Estado do Tocantins (SANEATINS) email: anachristinasilveira@gmail.com

2 Engenheiro Civil pela UNITAU, mestre em Ciências Cartográficas pela FCT/UNESP e doutor em Engenharia Química na área de Poluição, e preservação ambiental pela Universidade Estadual de Maringá (UEM). Professor da Universidade Federal do Tocantins no curso de Engenharia Ambiental. e-mail: rmontanhini@uft.edu.br

${ }^{3}$ Doutoranda no Programa de Pós-Graduação em Geografia UNESP - Universidade Estadual Paulista, Presidente Prudente e Doutoranda no Programa de Pós-Graduação em Arquitetura e Urbanismo da Universidade Presbiteriana Mackenzie. E-mail: sandra@sigmaambiente.com
} 
Palavras-Chave: Saneamento. Estação de tratamento de água ETA. Floculação e decantação.

\section{INTRODUÇÃO}

O conhecimento das características do escoamento nas unidades de tratamento é de grande importância para elaboração do projeto de novas estações e na avaliação e proposição de melhorias em estações existentes. Freqüentemente é observado que grande parte do mau funcionamento de Estações de Tratamento de Água (ETAs) está ligada a problemas hidráulicos nas unidades, relacionados principalmente à divisão desigual de vazão entre diferentes módulos de tratamento; curtos circuitos hidráulicos; zonas mortas e características inadequadas do meio filtrante (Di Bernardo et al., (2011).

A divisão desigual da vazão pode sobrecarregar um ou mais módulos, devido à maior vazão que escoa por eles. A existência de curto circuitos hidráulicos e de zonas mortas diminui consideravelmente o tempo de residência nas unidades. Devem ser consideradas ainda as características do escoamento da unidade - se do tipo pistão ou se mistura completa. A seqüência de aplicação e o local de introdução de produtos químicos, o gradiente de velocidade na mistura rápida e o tempo de residência nas unidades merecem atenção especial, dada a influência que exercem na coagulação e conseqüentemente, no desempenho das unidades subseqüentes.

Com o propósito de obter um diagnóstico real do funcionamento hidrodinâmico das unidades, foram desenvolvidas diversas técnicas investigativas, as quais foram aprimoradas ao longo dos anos, dentre elas o emprego de traçadores. Os traçadores são produtos químicos (não reativos) adicionados a água para monitoramento da sua concentração na água afluente ou efluente das unidades, porque para onde é encaminhado o traçador, é para onde a água escoa. As substâncias mais comuns empregadas como traçadores em estudos nas Estações de Tratamento de Água são o cloreto de sódio e cálcio.

Tendo em vista a importância dos estudos hidrodinâmicos em estações de tratamento de água, o presente trabalho buscou estudar o funcionamento da Estação de tratamento de água ETA 006 na cidade de Palmas-TO. A referida ETA é operada pela Companhia de Saneamento do Tocantins - SANEATINS e está em operação desde 1999. A tecnologia de tratamento é de ciclo completo e funciona atualmente com uma vazão de 
$500 \mathrm{~L} / \mathrm{s}$ tendo capacidade total para $700 \mathrm{~L} / \mathrm{s}$. A estação é responsável pela maior parte do abastecimento de Palmas, atendendo mais de $60 \%$ da população.

A água tratada na ETA 006 é captada no ribeirão Taquarussu, um pequeno manancial cuja nascente fica dentro do município de Palmas. Porém, a vazão do manancial nos últimos anos vem diminuindo consideravelmente, fazendo com que a Companhia de Saneamento avalie outra de fonte de abastecimento para Palmas.

Assim, devido à necessidade de reforma/ampliação da estação, e uma possível mudança de captação da água bruta, foi realizado o estudo hidrodinâmico na ETA 006, avaliando principalmente o funcionamento das unidades de floculação e decantação.

\section{MATERIAIS E MÉTODOS}

Para realização do estudo foi adotado a metodologia recomenda por (Di Bernardo e Dantas, 2005), com supervisão da empresa Hidrosan Engenharia. A Figura 1 mostra imagem da ETA 006, com as unidades de tratamento e a captação.

Figura 1: Imagem aérea da ETA 006 - Fonte: adaptado de SANEATINS

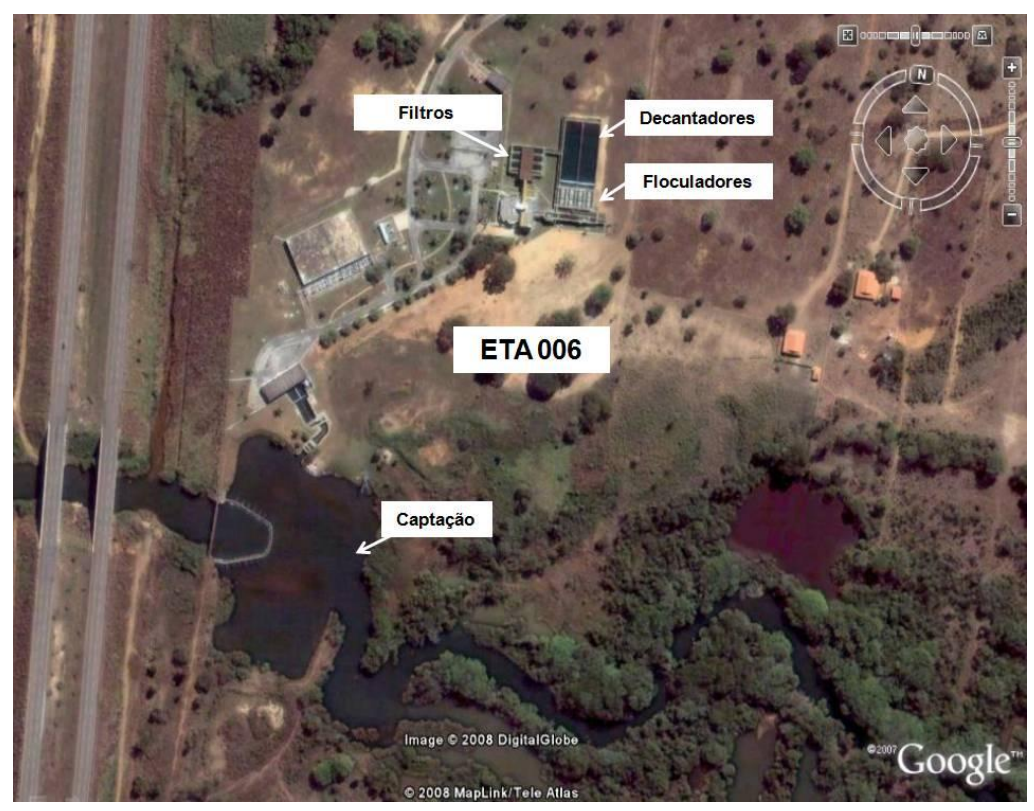




\subsection{Análise do comportamento hidrodinâmico da ETA}

A análise do comportamento hidrodinâmico da ETA foi feita através de quatro ensaios utilizando solução de cloreto de sódio ( $\mathrm{NaCl}$ - sal de cozinha) como traçador, com os objetivos de determinar a vazão veiculada na ETA, a vazão veiculada para cada módulo, o comportamento hidrodinâmico de uma unidade de floculação e o comportamento hidrodinâmico de um decantador.

\subsubsection{Aferição da vazão tratada na ETA}

Este ensaio teve como objetivo medir a vazão de trabalho na ETA com o uso do traçador e comparar com a medição realizada no canal Parshall. A solução de cloreto de sódio foi aplicada de forma contínua no inicio do canal de chegada da água bruta na ETA, e foram coletadas amostras antes do canal de divisão de vazão para os módulos de decantação/floculação. A solução foi preparada misturando-se $90 \mathrm{~kg}$ de sal (cloreto de sódio) num volume total de 447,69 L de água, sendo que a concentração de cloreto de sódio resultou de 201,7 $\mathrm{g} / \mathrm{L}$, conforme as equações a seguir:

$$
C m=\frac{m_{\text {sal }}}{V} \quad C m=\frac{90.000 \mathrm{~g}}{447,69 \mathrm{~L}} \quad C m=201,07 \mathrm{~g} / \mathrm{L}
$$

Considerando que a relação entre as massas molares do cloreto e o cloreto de sódio, em massa por massa, é de $0,607(35,5 / 58,5)$, o que resulta na concentração de $122,05 \mathrm{~g} / \mathrm{L}$ de cloretos na solução aplicada.

$$
C t=C m . f \quad C t=201,07 \times 0,607 \quad C t=122,05 \mathrm{~g} / \mathrm{L}
$$

A solução de cloreto de sódio foi aplicada com a vazão de 0,02995 L/s.

2.2.2 Divisão de vazão afluente a cada módulo de floculação/decantação, $Q=500 \mathrm{~L} / \mathrm{s}$ 
Para realização deste ensaio, a solução de cloreto de sódio foi aplicada na forma de pulso no inicio do canal de chegada da água bruta na ETA, e foram coletadas amostras nos canais que conduzem a água coagulada para os módulos de floculação/decantação, com o objetivo de determinar-se a vazão afluente a cada um dos módulos.

A solução foi preparada misturando-se $44 \mathrm{~kg}$ de sal num volume total de $220 \mathrm{~L}$ de água, sendo que a concentração total de cloreto de sódio resultou em 200,0 g/L, conforme as equações a seguir:

$$
C m=\frac{m_{\text {sal }}}{V} \quad C m=\frac{44.000 \mathrm{~g}}{220,0 \mathrm{~L}} \quad C m=200,0 \mathrm{~g} / \mathrm{L}
$$

Considerando que a relação entre as massas molares do cloreto e o cloreto de sódio, em massa por massa, é de 0,607 (35,5/58,5), resulta na concentração de 121,40 g/L de cloretos na solução aplicada.

$$
C t=C m \cdot f \quad C t=200,00.0,607 \quad C t=121,4 \mathrm{~g} / \mathrm{L}
$$

\subsubsection{Estudo hidrodinâmico do floculador e do decantador para $Q=350 \mathrm{~L} / \mathrm{s}$}

Neste ensaio foi avaliado apenas 01 módulo floculação/decantação com vazão de $350 \mathrm{~L} / \mathrm{s}$. A solução de cloreto de sódio foi preparada adicionando-se $44 \mathrm{~kg}$ de cloreto de sódio em uma bombona contendo 220 litros de água, aproximadamente. A solução foi adicionada na água afluente à unidade de floculação, logo após a divisão de vazão entre os módulos de floculação/decantação, como mostra a Figura 6.

Foram coletadas amostras na saída do floculador em três pontos e misturadas para representar a saída dessa unidade. Também foram feitas coletas na saída do decantador e da água bruta. 


\section{RESULTADOS E DISCUSSÕES}

\subsection{Resultados da aferição da vazão tratada na ETA}

No Quadro 1 têm-se o tempo de coleta, a vazão de aplicação da solução de cloreto de sódio (q), a concentração de cloretos na água bruta (Ca), a concentração de cloretos na água coletada (C), a concentração da solução do traçador (Ct), e a Vazão determinada pela equação a seguir:

$$
Q=\frac{q \cdot(C t-C)}{(C-C a)}
$$

Nota-se no Quadro 1 que os valores de vazão (Q) em vermelho não são representativos e foram desconsiderados, pois provavelmente ainda não havia ocorrido a estabilização da concentração de cloreto de sódio na água coletada, resultando vazões que não representam o que estava ocorrendo na ETA.

Quadro 1: Coletas, Concentração de cloretos e vazão tratada na ETA.

\begin{tabular}{|c|c|c|c|c|c|}
\hline $\begin{array}{c}\text { TEMPO } \\
(\mathrm{min})\end{array}$ & $\mathrm{q}(\mathrm{L} / \mathrm{s})$ & $\begin{array}{c}\mathrm{Ca} \\
(\mathrm{mg} / \mathrm{L})\end{array}$ & $\begin{array}{c}\mathrm{C} \\
(\mathrm{mg} / \mathrm{L})\end{array}$ & $\mathrm{C}-\mathrm{Ca}$ & $\mathrm{Q}(\mathrm{L} / \mathrm{s})$ \\
\hline 0 & 0,2995 & 2,45 & 1,96 & $-0,49$ & $74.581,9$ \\
\hline 0,5 & 0,2995 & 2,45 & 1,96 & $-0,49$ & $74.581,9$ \\
\hline 1 & 0,2995 & 2,45 & 2,45 & 0,00 & 0,0 \\
\hline 1,5 & 0,2995 & 2,45 & 2,45 & 0,00 & 0,0 \\
\hline 2 & 0,2995 & 2,45 & 13,23 & 10,78 & $3.389,8$ \\
\hline 3 & 0,2995 & 2,45 & 59,29 & 56,84 & 642,6 \\
\hline 4 & 0,2995 & 2,45 & 69,69 & 67,24 & 543,2 \\
\hline 5 & 0,2995 & 2,45 & 88,20 & 85,75 & 425,9 \\
\hline
\end{tabular}




\begin{tabular}{|c|c|c|c|c|c|}
\hline 6 & 0,2995 & 2,45 & 93,59 & 91,14 & 400,7 \\
\hline 7 & 0,2995 & 2,45 & 98,49 & 96,04 & 380,2 \\
\hline 8 & 0,2995 & 2,45 & 100,45 & 98,00 & 372,6 \\
\hline 8,5 & 0,2995 & 2,45 & 99,47 & 97,02 & 376,4 \\
\hline 10 & 0,2995 & 2,45 & 106,82 & 104,37 & 349,8 \\
\hline 12 & 0,2995 & 2,45 & 103,39 & 100,94 & 361,7 \\
\hline 14 & 0,2995 & 2,45 & 99,47 & 97,02 & 376,4 \\
\hline 16 & 0,2995 & 2,45 & 99,47 & 97,0 & 376,4 \\
\hline & & & & Média & 370,5 \\
\hline
\end{tabular}

A vazão determinada pelo medidor de vazão no vertedor Parshall da ETA, está mostrada no Quadro 2. Observa-se que a vazão determinada pelo vertedor Parshall está de acordo com a vazão obtida através da aplicação contínua da solução de cloreto.

Quadro 2: Vazão determinada pela lâmina do vertedor Parshall

\begin{tabular}{|c|c|}
\hline Horário & $\begin{array}{c}\text { Vazão } \\
\text { monitorada } \\
\text { (L/s) }\end{array}$ \\
\hline $9: 00$ & 361,0 \\
\hline $10: 00$ & 369,0 \\
\hline $11: 00$ & 370,0 \\
\hline
\end{tabular}

3.2 - Resultados da divisão de vazão afluente a cada módulo de floculação/decantação, $Q=500 \mathrm{~L} / \mathrm{s}$.

No Quadro 3 são mostrados os tempos de coleta, a concentração de cloretos na água bruta ( $\mathrm{Ca}$ ) e a concentração de cloretos na água coletada para os canais 1 e 2 (C1 e $\mathrm{C} 2$ ): 
Quadro 3: Tempo de coleta e concentração de cloretos.

\begin{tabular}{|c|c|c|c|c|c|c|c|}
\hline \multirow{2}{*}{$\begin{array}{l}\text { TEMPO } \\
\text { (min) }\end{array}$} & \multirow{2}{*}{$\begin{array}{c}\mathrm{Ca} \\
(\mathrm{mg} / \mathrm{L})\end{array}$} & \multicolumn{6}{|c|}{ AMOSTRAS (mg/L) } \\
\hline & & C 1 & $\mathrm{C} 1-\mathrm{Ca}$ & $\begin{array}{c}\text { (C1- } \\
\text { Ca). } \square \square t\end{array}$ & C 2 & C2 - Ca & $\begin{array}{c}\text { (C2- } \\
\text { Ca). } \square \square t\end{array}$ \\
\hline 0 & 2,45 & 3,43 & 0,98 & 0,25 & 2,94 & 0,49 & 0,12 \\
\hline 0,5 & 2,45 & 2,45 & 0,00 & 0,00 & 3,43 & 0,98 & 0,49 \\
\hline 1 & 2,45 & 2,45 & 0,00 & 0,00 & 8,82 & 6,37 & 3,19 \\
\hline 1,5 & 2,45 & 69,09 & 66,64 & 33,32 & 309,19 & 306,74 & 153,37 \\
\hline 2 & 2,45 & 362,11 & 359,66 & 179,83 & 117,60 & 115,15 & 57,58 \\
\hline 2,5 & 2,45 & 342,20 & 339,75 & 169,88 & 49,49 & 47,04 & 23,52 \\
\hline 3 & 2,45 & 338,10 & 335,65 & 251,74 & 41,16 & 38,71 & 29,03 \\
\hline 4 & 2,45 & 115,15 & 112,70 & 112,70 & 30,87 & 28,42 & 28,42 \\
\hline 5 & 2,45 & 40,67 & 38,22 & 38,22 & 6,86 & 4,41 & 4,41 \\
\hline 6 & 2,45 & 9,80 & 7,35 & 5,51 & 2,45 & 0,00 & 0,00 \\
\hline 6,5 & 2,45 & 4,90 & 2,45 & 1,23 & 1,47 & 0,00 & 0,00 \\
\hline 7 & 2,45 & 3,43 & 0,98 & 0,49 & 4,41 & 0,00 & 0,00 \\
\hline 7,5 & 2,45 & 3,92 & 0,00 & 0,00 & 1,96 & 0,00 & 0,00 \\
\hline 8,5 & 2,45 & 2,45 & 0,00 & 0,00 & 1,47 & 0,00 & 0,00 \\
\hline 10 & 2,45 & 2,94 & 0,00 & 0,00 & 1,96 & 0,00 & 0,00 \\
\hline 12 & 2,45 & 4,41 & 0,00 & 0,00 & 0,00 & 0,00 & 0,00 \\
\hline & & \multicolumn{2}{|c|}{$\square(\mathbf{C 1}-\mathbf{C a}) \cdot \square \square \mathbf{t}=$} & 793,16 & \multicolumn{2}{|c|}{$\square(\mathbf{C 2}-\mathbf{C a}) \cdot \square \square \mathbf{t}=$} & 300,16 \\
\hline
\end{tabular}

A vazão alfuente determinada pelo medidor de vazão no vertedor Parshall da ETA durante o ensaio era de $500 \mathrm{~L} / \mathrm{s}$. A vazão encaminhada para cada módulo da ETA foi determinada através da proporção, em massa de traçador, em para cada módulo. $O$ cálculo da vazão veiculada para cada módulo foi realizado através da equação:

$$
Q=500 \cdot \frac{\sum(C i-C a) \cdot \Delta t}{\sum[(C 1-C a)+(C 2-C a)] \cdot \Delta t}
$$

De acordo com a equação, as vazões veiculadas para o cada módulo da ETA estão mostradas no Quadro 4.

Quadro 4: Vazão veiculada para cada módulo de floculador/decantador.

\begin{tabular}{|c|c|}
\hline $\begin{array}{c}\text { Módulo de } \\
\text { floculador/decantador }\end{array}$ & $\begin{array}{c}\text { Vazão } \\
\text { calculada } \\
\text { (L/s) }\end{array}$ \\
\hline Módulo 01 & 362,74 \\
\hline Módulo 02 & 137,26 \\
\hline Total & $\mathbf{5 0 0 , 0}$ \\
\hline
\end{tabular}


Somando as concentrações de cloretos, multiplicados pelo intervalo de tempo e a respectiva vazão, de cada módulo, realizando a conversão para a massa de cloreto de sódio (1,65 vezes), considerando um teor de pureza de 95\%, tem-se que no total foram recuperados $35 \mathrm{~kg}$ de cloreto de sódio, dos $44 \mathrm{~kg}$ aplicados. A recuperação de apenas $80 \%$ da massa de cloreto de sódio aplicada deve-se provavelmente a erros de análise de cloretos, tanto na água bruta quanto nas amostras coletadas.

\subsection{Resultados do Estudo hidrodinâmico do floculador para $Q=350 \mathrm{~L} / \mathrm{s}$}

As características das unidades de floculação são:

Comprimento - 12,00 m

Largura - 12,00 m

Profundidade útil - 3,50 m

Volume $-504,00 \mathrm{~m}^{3}$

№ de câmaras - 04 unidades

Vazão de teste - $350 \mathrm{~L} / \mathrm{s}$

Tempo de detenção (To) - 24 minutos

Concentração de cloreto (Co) - 87,3 mg/L

Quadro 5: Concentração de cloretos das amostras coletadas na saída da unidade de floculação da ETA.

\begin{tabular}{|c|c|c|c|c|c|c|}
\hline \multirow{2}{*}{$\begin{array}{c}\text { TEMPO } \\
\text { (min) }\end{array}$} & \multirow{2}{*}{$\begin{array}{c}\mathrm{Ca} \\
(\mathrm{mg} / \mathrm{L})\end{array}$} & \multicolumn{4}{|c|}{ AMOSTRAS (mg/L) } & \multirow{2}{*}{$\begin{array}{r}\mathrm{Cm}-\mathrm{Ca} \\
(\mathrm{mg} / \mathrm{L})\end{array}$} \\
\hline & & $\begin{array}{c}\text { Comporta } \\
1\end{array}$ & \begin{tabular}{|c|} 
Comporta \\
2
\end{tabular} & \begin{tabular}{|c|} 
Comporta \\
3
\end{tabular} & $\begin{array}{c}\mathrm{C} \\
\text { mistura }\end{array}$ & \\
\hline 0 & 2,45 & 3,43 & 3,43 & 4,90 & 3,92 & 1,47 \\
\hline 3 & 2,45 & 19,11 & 26,95 & 11,27 & 19,11 & 16,66 \\
\hline 6 & 2,45 & 30,38 & 17,15 & 18,13 & 21,89 & 19,44 \\
\hline 9 & 2,45 & 36,26 & 32,83 & 40,61 & 36,57 & 34,12 \\
\hline 12 & 2,45 & 47,53 & 50,96 & 49,98 & 49,49 & 47,04 \\
\hline
\end{tabular}




\begin{tabular}{|c|c|c|c|c|c|c|}
15 & 2,45 & 60,27 & 53,90 & 55,37 & 56,51 & 54,06 \\
\hline 18 & 2,45 & 49,00 & 53,41 & 51,94 & 51,45 & 49,00 \\
\hline 21 & 2,45 & 47,04 & 47,04 & 48,51 & 47,53 & 45,08 \\
\hline 24 & 2,45 & 40,18 & 42,14 & 40,67 & 41,00 & 38,55 \\
\hline 30 & 2,45 & 25,48 & 24,99 & 28,42 & 26,30 & 23,85 \\
\hline 40 & 2,45 & 10,78 & 18,62 & 13,72 & 14,37 & 11,92 \\
\hline 50 & 2,45 & 5,88 & 6,86 & 5,88 & 6,21 & 3,76 \\
\hline 60 & 2,45 & 1,96 & 2,94 & 4,41 & 3,10 & 0,65 \\
\hline 75 & 2,45 & 4,41 & 6,37 & 5,39 & 5,39 & 2,94 \\
\hline 90 & 2,45 & 1,96 & 1,47 & 1,47 & 1,63 & 0 \\
\hline 120 & 2,45 & 0,98 & 1,96 & 2,45 & 1,80 & 0 \\
\hline 154 & 2,45 & 0,49 & 1,96 & 0,00 & 0,82 & 0 \\
\hline
\end{tabular}

Os valores em vermelho foram desconsiderados nos cálculos, pois estes valores resultaram num valor de "Cm-Ca" negativo, ou seja, a concentração de cloretos da água bruta foi maior do que a da água coletada. Para os cálculos, os valores de "Cm-Ca" para os tempos de 90,120, 154 minutos foram considerados iguais a zero.

Foram calculados os parâmetros para efetuar a análise do comportamento hidráulico da unidade de floculação, considerando que o volume total das câmaras de floculação é de $504 \mathrm{~m}^{3}$, que corresponde à um tempo teórico de detenção ( $\mathrm{t}_{\mathrm{o}}$ ) de 24 minutos para a vazão de $350 \mathrm{~L} / \mathrm{s}$.

Os itens calculados são:

$\Sigma \mathrm{C}_{\mathrm{i}} \cdot \Delta \mathrm{t}=$ somatório da concentração de cloretos multiplicado pelo intervalo de tempo correspondente.

$F_{(t)}=$ fração dos elementos de fluido que sai da unidade em um tempo $t$. 


$$
F_{(t)}=\frac{\sum_{i=1}^{n} C i \cdot \Delta t}{\sum_{i=1}^{m} C i \cdot \Delta t}
$$

$1-F_{(t)}=$ fração do escoamento que permanece por um tempo maior que $t$ na unidade.

Quadro 6: Valores de $\Sigma C, F_{(t)}$ e 1- $F_{(t)}$ para a Unidade de Floculação $(Q=350 \mathrm{~L} / \mathrm{s})$.

\begin{tabular}{|c|c|c|c|c|c|}
\hline $\begin{array}{c}\mathbf{t} \\
\text { (min) }\end{array}$ & $\mathbf{t} / \mathbf{t o}$ & $\begin{array}{c}\mathbf{C m}-\mathbf{C a} \\
\text { (mg/L) }\end{array}$ & $\begin{array}{c}\sum \mathbf{C . \Delta t} \\
\text { (mg/L) }\end{array}$ & $\mathbf{F t}$ & $\mathbf{1 - F t}$ \\
\hline 0 & 0,00 & 0,98 & 1,47 & 0,00 & 1,00 \\
\hline 3 & 0,13 & 17,15 & 52,92 & 0,04 & 0,96 \\
\hline 6 & 0,25 & 21,56 & 117,60 & 0,09 & 0,91 \\
\hline 9 & 0,38 & 35,28 & 223,44 & 0,18 & 0,82 \\
\hline 12 & 0,50 & 47,53 & 366,03 & 0,29 & 0,71 \\
\hline 15 & 0,63 & 49,98 & 515,97 & 0,40 & 0,60 \\
\hline 18 & 0,75 & 47,04 & 657,09 & 0,51 & 0,49 \\
\hline 21 & 0,88 & 42,00 & 783,09 & 0,61 & 0,39 \\
\hline 24 & 1,00 & 40,18 & 963,90 & 0,76 & 0,24 \\
\hline 30 & 1,25 & 25,00 & 1163,90 & 0,91 & 0,09 \\
\hline 40 & 1,67 & 7,84 & 1242,30 & 0,97 & 0,03 \\
\hline 50 & 2,08 & 3,43 & 1276,60 & 1,00 & 0,00 \\
\hline 60 & 2,50 & 0,00 & 1276,60 & 1,00 & 0,00 \\
\hline
\end{tabular}

Na Figura 2 foram lançados os valores de $F(t)$ em função de $t / t_{0}$ ( $t$ é o tempo de coleta de amostra e to é o tempo teórico de detenção na unidade, igual a $24 \mathrm{~min}$.). A partir dessa figura tem-se que $75 \%$ do traçador sai antes do tempo teórico de detenção e praticamente todo o traçador sai da unidade em até duas vezes o tempo teórico de detenção. 
Figura 2: Fração do tempo teórico de detenção $x \mathrm{~F}(\mathrm{t})$.

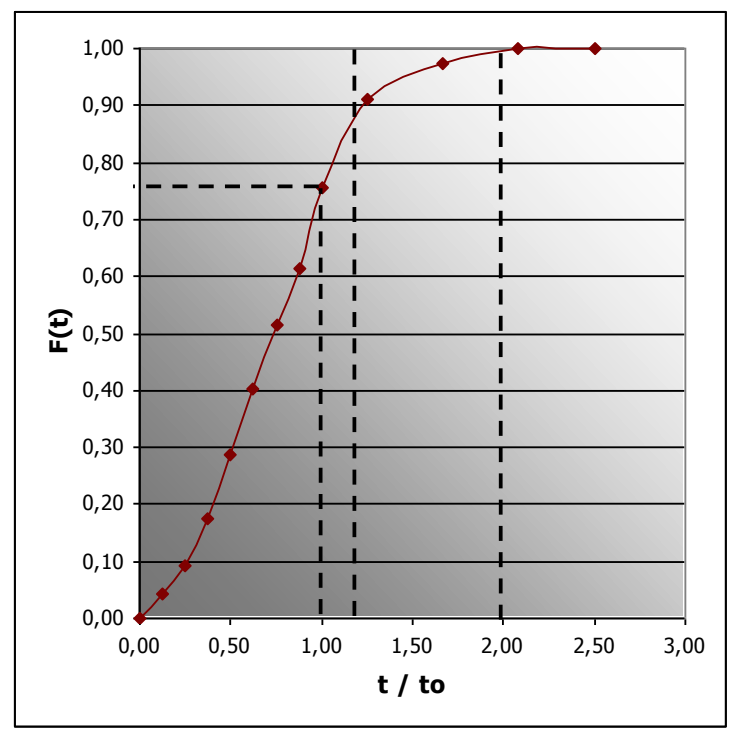

Lançando os valores de 1-F(t) em escala logarítmica no eixo das ordenadas e os valores de $t / t_{0}$ no eixo das abscissas, construiu-se a Figura 3 , na qual se obtém:

$$
\begin{aligned}
& \text { p. }(1-\mathrm{m})=0,65 \\
& \operatorname{tg} \alpha=\frac{t_{0}}{t_{1}-t_{2}}=\frac{-\log e}{(1-p) \cdot(1-m)}=\frac{1}{-0,57}
\end{aligned}
$$

Onde $p$ é a fração de escoamento do tipo pistão; (1-p) é a fração do escoamento do tipo mistura completa; e $\mathrm{m}$ é a porcentagem do volume correspondente às zonas mortas.

Resolvendo as equações acima resulta:

$$
\begin{aligned}
& p=0,70 \\
& (1-p)=0,30 \\
& m=0,086
\end{aligned}
$$


Figura 3: Fração do tempo teórico de detenção em função de 1- F(t) (em escala logarítmica).

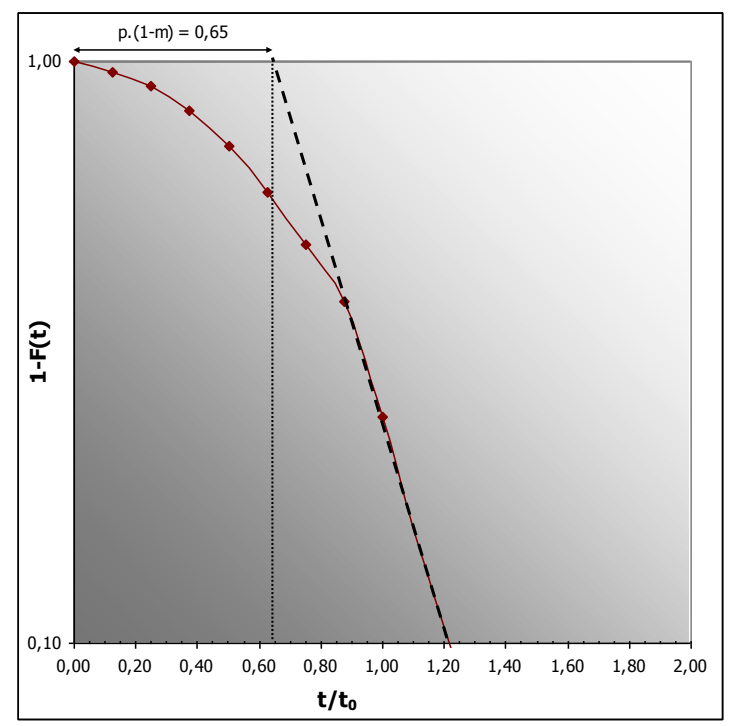

Nessas condições concluiu-se que a fração do escoamento tipo pistão (p) é de $70 \%$, a parcela de escoamento do tipo mistura completa (1-p) é de $30 \%$ e a fração do volume da unidade correspondente às zonas mortas é de $8,6 \%$

\subsection{Resultados do Estudo hidrodinâmico do decantador para Q=350 L/s}

As características das unidades de decantação são:

Comprimento $-40,00 \mathrm{~m}$

Largura - 12,00 m

Profundidade útil $-4,20 \mathrm{~m}$

Volume - 2.016,00 $\mathrm{m}^{3}$

№ de calhas -08 unidades

Vazão de teste $-350 \mathrm{~L} / \mathrm{s}$

Tempo de detenção (To) -96 minutos

Concentração de cloreto (Co) $-34,92 \mathrm{mg} / \mathrm{L}$ 
Neste item foram calculados os parâmetros para análise do comportamento hidráulico da unidade de decantação. O volume total do decantador é de $2.016 \mathrm{~m}^{3}$, que corresponde à um tempo teórico de detenção $\left(\mathrm{t}_{\mathrm{o}}\right)$ de 96 minutos para a vazão de $350 \mathrm{~L} / \mathrm{s}$.

Os itens calculados são:

$\Sigma \mathrm{C}_{\mathrm{i} .} . \Delta \mathrm{t}=$ somatório da concentração de cloretos multiplicado pelo intervalo de tempo correspondente.

$F_{(t)}=$ fração dos elementos de fluido que sai da unidade em um tempo $t$.

$$
F_{(t)}=\frac{\sum_{i=1}^{n} C i \cdot \Delta t}{\sum_{i=1}^{m} C i \cdot \Delta t}
$$

$1-F_{(t)}=$ fração do escoamento que permanece por um tempo maior que $t$ na unidade.

Quadro 7: $\Sigma C, F_{(t)}$ e 1-F(t) para as Unidades de Decantação da ETA.

\begin{tabular}{|c|c|c|c|c|c|}
\hline $\begin{array}{c}\mathrm{t} \\
(\mathrm{min})\end{array}$ & t/to & $\begin{array}{l}\text { Cm-Ca } \\
\text { (mg/L) }\end{array}$ & $\underset{(\mathrm{mg} / \mathrm{L})}{\sum \mathrm{C} . \Delta \mathrm{t}}$ & $\mathbf{F t}$ & 1-Ft \\
\hline 30 & 0,86 & 3,26 & 0,04 & 0,04 & 0,96 \\
\hline 60 & 1,72 & 15,18 & 0,32 & 0,29 & 0,71 \\
\hline 75 & 2,15 & 10,74 & 0,45 & 0,40 & 0,60 \\
\hline 90 & 2,58 & 7,54 & 0,54 & 0,49 & 0,51 \\
\hline 105 & 3,01 & 6,07 & 0,62 & 0,55 & 0,45 \\
\hline 120 & 3,44 & 3,94 & 0,67 & 0,60 & 0,40 \\
\hline 135 & 3,87 & 5,09 & 0,73 & 0,65 & 0,35 \\
\hline 150 & 4,30 & 5,64 & 0,80 & $\begin{array}{l}0,71 \\
\end{array}$ & 0,29 \\
\hline 165 & 4,73 & 5,31 & 0,87 & 0,77 & 0,23 \\
\hline 180 & 5,15 & 4,20 & 0,92 & 0,82 & 0,18 \\
\hline 195 & 5,58 & 3,42 & 0,96 & 0,86 & 0,14 \\
\hline 210 & 6,01 & 3,13 & 1,00 & 0,89 & $\mathbf{0 , 1 1}$ \\
\hline 225 & 6,44 & 2,60 & 1,03 & 0,92 & 0,08 \\
\hline 240 & 6,87 & 2,31 & 1,07 & 0,96 & 0,04 \\
\hline 270 & 7,73 & 1,91 & 1,13 & 1,00 & 0,00 \\
\hline 300 & 8,59 & 0,00 & 1,19 & 1,00 & 0,00 \\
\hline 325 & 9,31 & 0,00 & 1,27 & 1,00 & 0,00 \\
\hline
\end{tabular}

Nota-se que para as amostras de água coletadas na saída do decantador são correspondentes ao tempo de detenção do floculador somado ao do decantador.

A partir dos dados do quadro 7 apenas para decantador foram realizadas as análises dessa unidade:

$$
\text { p. }(1-m)=0,50
$$




$$
\operatorname{tg} \alpha=\frac{t_{0}}{t_{1}-t_{2}}=\frac{-\log e}{(1-p) \cdot(1-m)}=\frac{1}{-1,75}
$$

Onde $p$ é a fração de escoamento do tipo pistão; (1-p) é a fração do escoamento do tipo mistura completa; e $m$ é a porcentagem do volume correspondente às zonas mortas.

Figura 4: Fração do Tempo Teórico de Detenção em função de 1- F(t) (em escala logarítmica).

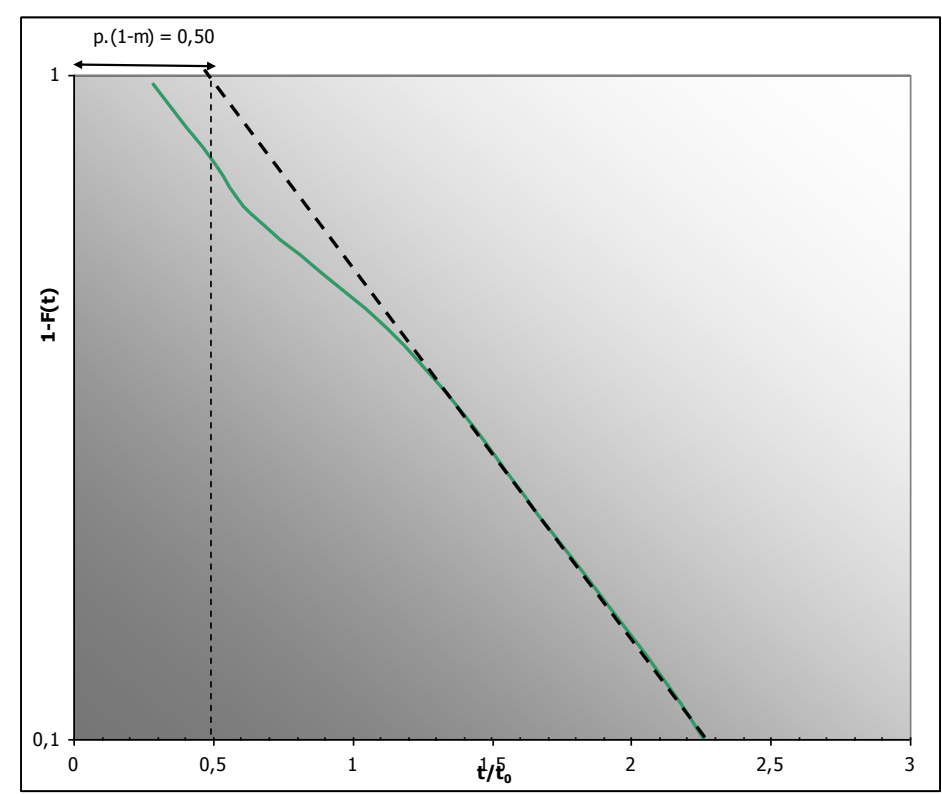

Resolvendo as equações acima temos que:

$$
\begin{aligned}
& p=0,40 \\
& (1-p)=0,60 \\
& m=-0,26 \text { (valor negativo) }
\end{aligned}
$$

$\mathrm{Na}$ Figura 5 foram lançados os valores de $\mathrm{F}(\mathrm{t})$, considerando apenas o decantador, em função de $t / t_{0}$. A partir dessa figura tem-se que $53 \%$ do traçador sai antes do tempo teórico de detenção e que cerca de $95 \%$ do traçador sai da unidade em até duas vezes e meia o tempo teórico de detenção dessa unidade. 
Figura 5: Fração do Tempo Teórico de Detenção em Função de F(t).

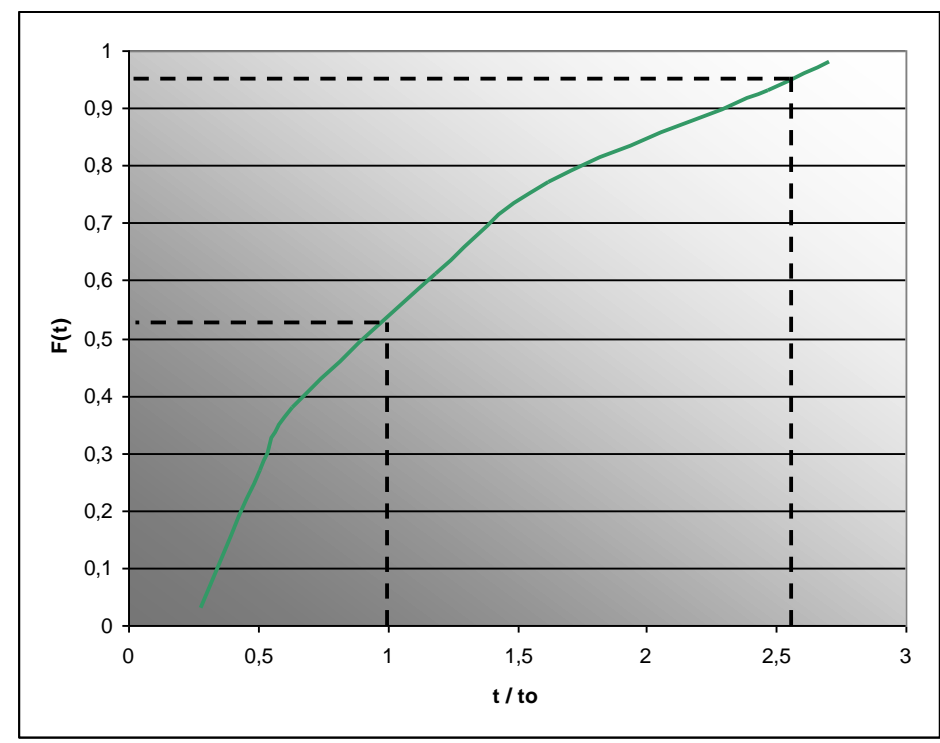

Resolvendo o equacionamento para os valores encontrados na Figura 5, o valor da porcentagem do volume do decantador correspondente a zonas mortas é negativo, que não tem representação prática. Realizando os cálculos para obtenção da massa de cloreto de sódio total recuperada pelos ensaios, tem-se que foram recuperados $56 \mathrm{~kg}$, sendo que foram aplicados apenas $44 \mathrm{~kg}$ de cloreto de sódio no ensaio. Nessas condições, é possível que a determinação de cloretos na água não tinha sido precisa ou que podem ter ocorridos contaminações das amostras, diluições, determinação dos cloretos, ou na determinação de cloretos na água bruta durante o ensaio. Esta ultima possibilidade é provável de ter acontecido, por não zerar a concentração de cloretos após mais de 3 vezes o tempo de detenção e já ter sido recuperada a massa total de cloro de sódio utilizada no ensaio

\section{CONCLUSÕES}

A partir dos resultados obtidos nos ensaios realizados, podemos chegar as seguintes conclusões: 
- A divisão de vazão, por meio de comportas com passagem inferior, quando a vazão afluente a ETA foi de $500 \mathrm{~L} / \mathrm{s}$, resultou inadequada, com vazão de $362 \mathrm{~L} / \mathrm{s}$ para o módulo 01 e de $138 \mathrm{~L} / \mathrm{s}$ para o módulo 02

- Esta ineficiência na divisão de vazão pode estar provocando uma sobrecarga no decantador do módulo 1 , fato comprovado pela maior quantidade de lodo que é retirado deste decantador.

- Para a vazão de $350 \mathrm{~L} / \mathrm{s}$ em um único conjunto de floculação, o uso de traçador aplicado na forma de pulso indicou os seguintes resultados: fração do escoamento do tipo pistão $=70 \%$; fração do escoamento do tipo mistura completa $=30 \%$; fração do volume correspondente às zonas mortas $=8,6 \%$;

- Para a vazão de $350 \mathrm{~L} / \mathrm{s}$ em um único decantador, os resultados não foram coerentes, impossibilitando a análise hidrodinâmica da unidade;

- O estudo hidroninâmico para ETAs de ciclo completo mostrou ser uma ferramenta bastante prática e eficiente para se avaliar o funcionamento das unidades da estação.

\section{REFERÊNCIAS BIBLIOGRÁFICAS}

American Public Health Association, American Water Works Association and Water Environmental Federation. Standard Methods for the Examination of Water and Wastewater. 19th edition, Washington, USA. APHA, 1998.

DI BeRnARDO, L. ; DANTAS, A. D. B. Métodos e Técnicas de Tratamento de Água. 2. ed. São Carlos: Rima Editora, 2005. v. 2. 1584 p

DI BERNARDO, L.; DANTAS, A. D. B.; Voltan, P. E. N. Tratabilidade de Água e dos Resíduos gerados em Estações de Tratamento de Água. 1. ed. São Carlos: Editora LDiBe, 2011. v. 1. 454p.

HIDROSAN, Engenharia, Relatório 1 - Estudos de Avaliação do Desempenho da ETA 006 de Palmas.2009. 